International Mathematical Forum, Vol. 8, 2013, no. 40, 1985 - 1997

HIKARI Ltd, www.m-hikari.com

http://dx.doi.org/10.12988/imf.2013.311220

\title{
Variational Problems and Asymptotic Behaviors Associated with Liquid Crystals
}

\author{
Junichi Aramaki \\ Division of Science, Faculty of Science and Engineering \\ Tokyo Denki University \\ Hatoyama-machi, Saitama 350-0394, Japan \\ aramaki@mail.dendai.ac.jp
}

Copyright (C) 2013 Junichi Aramaki. This is an open access article distributed under the Creative Commons Attribution License, which permits unrestricted use, distribution, and reproduction in any medium, provided the original work is properly cited.

\begin{abstract}
The purpose of this paper is to give answers for the problems given by Pan [9] associated with the mathematical theory of liquid crystals for any bounded smooth domain, and the boundary data satisfying some condition.
\end{abstract}

Mathematics Subject Classification: 49J20, 58E20, 82D30

Keywords: variational problems, liquid crystals, asymptotic behavior of minimizers

\section{Introduction}

In this paper, we shall consider the variational problem associated with the mathematical theory of liquid crystals. Since we treat the Dirichlet problem for director field, we use the modified Landau-de Gennes energy functional (cf. de Gennes and Prost [6]).

$$
\begin{aligned}
\mathcal{E}[\psi, \boldsymbol{n}]=\int_{\Omega}\left\{\left|\nabla_{q \boldsymbol{n}} \psi\right|^{2}\right. & -\kappa^{2}|\psi|^{2}+\frac{\kappa^{2}}{2}|\psi|^{4} \\
& \left.+K_{1}(\operatorname{div} \boldsymbol{n})^{2}+K_{2}|\boldsymbol{n} \cdot \operatorname{curl} \boldsymbol{n}|^{2}+K_{3}|\boldsymbol{n} \times \operatorname{curl} \boldsymbol{n}|^{2}\right\} d x
\end{aligned}
$$


where $\Omega$ is a smooth bounded domain in $\mathbb{R}^{3}$ occupied by liquid crystal material, $\kappa, K_{i}(i=1,2,3)$ are positive constants called by the Ginzburg-Landau parameter and elastic coefficients, respectively and $q \in \mathbb{R}$ called by the wave number. We used the notation $\nabla_{q \boldsymbol{n}} \psi=\nabla \psi-i q \boldsymbol{n} \psi, i=\sqrt{-1}$. The state of liquid crystal can be described by a complex-valued function $\psi$ called order parameter and a unit vector field $\boldsymbol{n}$ called director field. $\psi=0$ means a nematic phase and $\psi \neq 0$ means a smectic phase. The functional

$$
\mathcal{F}[\boldsymbol{n}]=\int_{\Omega}\left\{K_{1}(\operatorname{div} \boldsymbol{n})^{2}+K_{2}|\boldsymbol{n} \cdot \operatorname{curl} \boldsymbol{n}|^{2}+K_{3}|\boldsymbol{n} \times \operatorname{curl} \boldsymbol{n}|^{2}\right\} d x
$$

is called the simplified Oseen-Frank energy functional for nematics and

$$
\mathcal{G}[\psi, \boldsymbol{n}]=\int_{\Omega}\left\{\left|\nabla_{q \boldsymbol{n}} \psi\right|^{2}-\kappa^{2}|\psi|^{2}+\frac{\kappa^{2}}{2}|\psi|^{4}\right\} d x
$$

is called the Ginzburg-Landau energy functional for smectics. We consider the functional $\mathcal{E}$ under the Dirichlet boundary condition for the director field $\boldsymbol{e}$. Let $W^{1,2}\left(\Omega, \mathbb{R}^{3}\right)$ be the usual Sobolev space of vector fields,

$$
W^{1,2}\left(\Omega . \mathbb{S}^{2}\right)=\left\{\boldsymbol{u} \in W^{1,2}\left(\Omega, \mathbb{R}^{3}\right) ;|\boldsymbol{u}(x)|=1 \text { a.e. in } \Omega\right\}
$$

and for the given field $\boldsymbol{e}: \partial \Omega \rightarrow \mathbb{S}^{2}$, we define

$$
W^{1,2}\left(\Omega, \mathbb{S}^{2}, \boldsymbol{e}\right)=\left\{\boldsymbol{u} \in W^{1,2}\left(\Omega, \mathbb{S}^{2}\right) ; \boldsymbol{u}=\boldsymbol{e} \text { on } \partial \Omega\right\}
$$

and

$$
\mathbb{W}(\Omega, \boldsymbol{e})=W^{1,2}(\Omega, \mathbb{C}) \times W^{1,2}\left(\Omega, \mathbb{S}^{2}, \boldsymbol{e}\right)
$$

Here we note that if $\boldsymbol{e}: \partial \Omega \rightarrow \mathbb{S}^{2}$ is Lipschitz, then $W^{1,2}\left(\Omega, \mathbb{S}^{2}, \boldsymbol{e}\right)$ is a non empty set (cf. Hardt et al. [8, Lemma 1.1]). Consider the following variational problem

$$
C_{d}\left(K_{1}, K_{2}, K_{3}, \kappa, q\right)=\inf _{(\psi, \boldsymbol{u}) \in \mathbb{W}(\Omega, \boldsymbol{e})} \mathcal{E}[\psi, \boldsymbol{u}]
$$

By the standard variational arguments (cf. [8], Pan [9]), we can show that if $\boldsymbol{e}$ is a smooth unit vector field on $\partial \Omega$ and $\Omega$ is a bounded smooth domain in $\mathbb{R}^{3}$, for any $K_{1}, K_{2}, K_{3}, \kappa>0, q \in \mathbb{R}$, the minimizers of the variational problem exist in $\mathbb{W}(\Omega, e)$.

We also consider the following variational problem for the Oseen-Frank energy

$$
N\left(K_{1}, K_{2}, K_{3}, \boldsymbol{e}\right)=\inf _{\boldsymbol{u} \in W^{1,2}\left(\Omega, \mathbb{S}^{2}, \boldsymbol{e}\right)} \mathcal{F}[\boldsymbol{u}]
$$


We can also show that $N\left(K_{1}, K_{2}, K_{3}, \boldsymbol{e}\right)$ is achieved. If we choose a minimizer $\boldsymbol{n} \in W^{1,2}\left(\Omega, \mathbb{S}^{2}, \boldsymbol{e}\right)$, then $(0, \boldsymbol{n})$ is a trivial critical point of $\mathcal{E}$ which corresponds with a nematic state. Aramaki [4] considered the behavior of minimizers when $K_{1}, K_{2}$ or $K_{3}$ tends to the infinity, along to the line of [9] in which the author treated the case where $K_{2}=K_{3}$. In the present paper, we also consider the case where $K_{2}=K_{3}$. Thus the Landau-de Gennes functional and the OseenFrank functional and the variational problems have the following forms.

$$
\mathcal{E}_{0}[\psi, \boldsymbol{n}]=\int_{\Omega}\left\{\left.\nabla_{q \boldsymbol{n}} \psi\right|^{2}-\kappa^{2}|\psi|^{2}+\frac{\kappa^{2}}{2}|\psi|^{4}+K_{1}(\operatorname{div} \boldsymbol{n})^{2}+K_{2}|\operatorname{curl} \boldsymbol{n}|^{2}\right\} d x,
$$

and

$$
C_{d}\left(K_{1}, K_{2}, \kappa, q\right)=\inf _{(\psi, \boldsymbol{n}) \in \mathbb{W}(\Omega, \boldsymbol{e})} \mathcal{E}_{0}[\psi, \boldsymbol{n}]
$$

For the modified Oseen-Frank energy functional becomes

$$
\mathcal{F}_{0}[\boldsymbol{n}]=\int_{\Omega}\left\{K_{1}(\operatorname{div} \boldsymbol{n})^{2}+K_{2}|\operatorname{curl} \boldsymbol{n}|^{2}\right\} d x
$$

and

$$
N\left(K_{1}, K_{2}, \boldsymbol{e}\right)=\inf _{\boldsymbol{n} \in W^{1,2}\left(\Omega, \mathbb{S}^{2}, \boldsymbol{e}\right)} \mathcal{F}_{0}[\boldsymbol{n}] .
$$

According to [9], define

$$
\mathbb{G}(\boldsymbol{e})=\mathbb{G}(\boldsymbol{e}, \Omega)=\left\{\phi \in W^{2,2}(\Omega) ;|\nabla \phi|=1 \text { a.e. in } \Omega, \nabla \phi=\boldsymbol{e} \text { on } \partial \Omega\right\} .
$$

If $\mathbb{G}(\boldsymbol{e}) \neq \emptyset$, we put

$$
G(\boldsymbol{e})=G(\boldsymbol{e}, \Omega)=\inf _{\phi \in \mathbb{G}(\boldsymbol{e})} \int_{\Omega}|\Delta \phi|^{2} d x .
$$

Then [9] got the following result.

Proposition 1.1. Assume that $\mathbb{G}(\boldsymbol{e}) \neq \emptyset$ and $\Omega$ is a smooth simply-connected domain in $\mathbb{R}^{3}$. Then

(i) $G(\boldsymbol{e})$ is achieved in $\mathbb{G}(\boldsymbol{e})$.

(ii) $\lim _{K_{2} \rightarrow \infty} N\left(K_{1}, K_{2}, \kappa, \boldsymbol{e}\right)=K_{1} G(\boldsymbol{e})$.

(iii) Let $\boldsymbol{u}_{j}$ be a minimizer of $N\left(K_{1}, K_{2}, \kappa, \boldsymbol{e}\right)$ for $K_{2}=K_{2}^{(j)}$ where $K_{2}^{(j)} \rightarrow$ $\infty$ as $j \rightarrow \infty$. Then there exist a subsequence $\left\{\boldsymbol{u}_{j_{k}}\right\}$ and $\phi \in \mathbb{G}(\boldsymbol{e})$ which is a minimizer of $G(\boldsymbol{e})$ such that $\boldsymbol{u}_{j_{k}} \rightarrow \nabla \phi$ strongly in $W^{1,2}\left(\Omega, \mathbb{R}^{3}\right)$ as $k \rightarrow \infty$.

Moreover define

$$
\mathbb{R}(\boldsymbol{e})=\mathbb{R}(\boldsymbol{e}, \Omega)=\left\{\boldsymbol{u} \in W^{1,2}\left(\Omega, \mathbb{S}^{2}, \boldsymbol{e}\right) ; \operatorname{div} \boldsymbol{u}=0 \text { a.e. in } \Omega\right\} .
$$

If $\mathbb{R}(\boldsymbol{e}) \neq \emptyset$, we put

$$
R(\boldsymbol{e})=R(\boldsymbol{e}, \Omega)=\inf _{\boldsymbol{u} \in \mathbb{R}(\boldsymbol{e})} \int_{\Omega}|\operatorname{curl} \boldsymbol{u}|^{2} d x .
$$

Then [9] also get 
Proposition 1.2. Assume that $\mathbb{R}(\boldsymbol{e}) \neq \emptyset$ and $\Omega$ is a smooth simply-connected domain in $\mathbb{R}^{3}$. Then

(i) $R(\boldsymbol{e})$ is achieved in $\mathbb{R}(\boldsymbol{e})$.

(ii) $\lim _{K_{1} \rightarrow \infty} N\left(K_{1}, K_{2}, \kappa, \boldsymbol{e}\right)=K_{2} R(\boldsymbol{e})$.

(iii) Let $\boldsymbol{u}_{j}$ be a minimizer of $N\left(K_{1}, K_{2}, \kappa, \boldsymbol{e}\right)$ for $K_{1}=K_{1}^{(j)}$ where $K_{1}^{(j)} \rightarrow$ $\infty$ as $j \rightarrow \infty$. Then there exist a subsequence $\left\{\boldsymbol{u}_{j_{k}}\right\}$ and $\boldsymbol{u} \in \mathbb{R}(\boldsymbol{e})$ which is a minimizer of $R(\boldsymbol{e})$ such that $\boldsymbol{u}_{j_{k}} \rightarrow \nabla \phi$ strongly in $W^{1,2}\left(\Omega, \mathbb{R}^{3}\right)$ as $k \rightarrow \infty$.

Using these facts, [9] got the asymptotic behavior of $C_{d}\left(K_{1}, K_{2}^{(j)}, \kappa, q\right)$ where $K_{2}^{(j)} \rightarrow \infty$ or $C_{d}\left(K_{1}^{(j)}, K_{2},, \kappa, q\right)$ where $K_{1}^{(j)} \rightarrow \infty$, and [4] extended the results to the case where one of $K_{1}, K_{2}$ and $K_{3}$ tends to the infinity.

Finally in the last section of the paper [9] the author proposed the following problems.

Problem 1. Find conditions on $\Omega$ and $\boldsymbol{e}$ such that $\mathbb{G}(\boldsymbol{e}, \Omega) \neq \emptyset$.

Problem 2. Find conditions on $\Omega$ and $\boldsymbol{e}$ such that $\mathbb{R}(\boldsymbol{e}, \Omega) \neq \emptyset$.

In this paper, we shall concern with these problems. We shall see that if $\Omega$ is a general bounded smooth domain in $\mathbb{R}^{3}$, and $\boldsymbol{e}$ has a some condition, then we shall give a necessary and sufficient condition to be $\mathbb{G}(\boldsymbol{e}, \Omega) \neq \emptyset$ or $\mathbb{R}(\boldsymbol{e}, \Omega) \neq \emptyset$.

\section{An equivalent condition to be $\mathbb{G}(e, \Omega) \neq \emptyset$}

Let $\Omega \subset \mathbb{R}^{3}$ be a bounded smooth domain and $e \in C^{1}\left(\partial \Omega, \mathbb{S}^{2}\right)$. According to Pan and Qi [10, Proposition 2.9], they proved that $\boldsymbol{e} \in C^{1}\left(\partial \Omega, \mathbb{R}^{3}\right)$ has a $C^{1}$ curl-free extension, that is to say, there exists $\phi \in C^{2}(\bar{\Omega})$ such that $\nabla \phi=\boldsymbol{e}$ on $\partial \Omega$ if and only if $\boldsymbol{e}_{\tau}=\nabla_{\tau} \psi$ for some $\psi \in C^{2}(\partial \Omega)$ where $\boldsymbol{e}_{\tau}$ is the tangential component of $\boldsymbol{e}: \boldsymbol{e}_{\tau}=\boldsymbol{e}-(\boldsymbol{e} \cdot \boldsymbol{\nu}) \boldsymbol{\nu}, \boldsymbol{\nu}$ is the unit outward normal vector field to $\partial \Omega$, and $\nabla_{\tau} \psi$ is the tangential component of $\nabla \psi$. Therefore a necessary condition to be $\mathbb{G}(\boldsymbol{e}) \neq \emptyset$ is

$$
\boldsymbol{e}_{\tau}=\nabla_{\tau} \psi \text { for some } \psi \in C^{2}(\partial \Omega) .
$$

In fact, if we assume (2.1), then the curl-free extension $\nabla \phi$ is constructed as follows. First we extend $\psi$ to be a function of $C^{2}(\bar{\Omega})$. Choose a unique solution $\zeta$ of the equation

$$
\begin{cases}\Delta^{2} \zeta=0 & \text { in } \Omega \\ \zeta=0, \frac{\partial \zeta}{\partial \boldsymbol{\nu}}=\boldsymbol{e} \cdot \boldsymbol{\nu}-\frac{\partial \psi}{\partial \boldsymbol{\nu}} & \text { on } \partial \Omega\end{cases}
$$

Then $\phi=\psi+\zeta$ gives a $C^{2}$ curl-free extension of $\boldsymbol{e}$.

Define

$$
\varphi_{0}=\phi=\psi, \quad \varphi_{1}=\frac{\partial \phi}{\partial \boldsymbol{\nu}}=\boldsymbol{e} \cdot \boldsymbol{\nu}
$$


on $\partial \Omega$. Thus the space

$$
W^{2,2}\left(\Omega, \varphi_{0}, \varphi_{1}\right)=\left\{\phi \in W^{2,2}(\Omega) ; \phi=\varphi_{0}, \frac{\partial \phi}{\partial \boldsymbol{\nu}}=\varphi_{1} \text { on } \partial \Omega\right\}
$$

is not an empty set. The minimizing problem (1.6) is closely related with the following variational problem.

$$
\begin{gathered}
J_{\varepsilon}[\phi]=\int_{\Omega}\left\{|\Delta \phi|^{2}+\frac{1}{\varepsilon^{2}}\left(1-|\nabla \phi|^{2}\right)^{2}\right\} d x, \\
c_{g}(\varepsilon)=\inf _{\phi \in W^{2,2}\left(\Omega, \varphi_{0}, \varphi_{1}\right)} J_{\varepsilon}[\phi] .
\end{gathered}
$$

We have the following theorem.

Theorem 2.1. Let $\Omega \subset \mathbb{R}^{3}$ be a bounded smooth domain in $\mathbb{R}^{3}$, and let $\boldsymbol{e} \in C^{1}\left(\partial \Omega, \mathbb{S}^{2}\right)$ satisfying (2.1). Moreover, let $\varphi_{0}, \varphi_{1}$ be functions on $\partial \Omega$ constructed from $\boldsymbol{e}$ as in (2.2). Then the following holds.

(i) For any $\varepsilon>0, c_{g}(\varepsilon)$ is achieved.

(ii) $\mathbb{G}(\boldsymbol{e}) \neq \emptyset$ if and only if $\left\{c_{g}(\varepsilon)\right\}_{\varepsilon>0}$ is uniformly bounded with respect to $\varepsilon>0$.

In this case, $c_{g}(\varepsilon) \rightarrow G(\boldsymbol{e})$ as $\varepsilon \rightarrow 0$, and for any minimizer $\phi_{\varepsilon}$ of $c_{g}(\varepsilon)$, there exist a subsequence $\left\{\phi_{\varepsilon_{j}}\right\}$ and $\phi \in \mathbb{G}(\boldsymbol{e})$ which is a minimizer of $G(\boldsymbol{e})$ such that $\phi_{\varepsilon_{j}} \rightarrow \phi$ strongly in $W^{2,2}(\Omega)$ as $\varepsilon_{j} \rightarrow 0$.

Proof. (i) For fixed $\varepsilon>0$, let $\left\{\phi_{j}\right\} \subset W^{2,2}\left(\Omega, \varphi_{0}, \varphi_{1}\right)$ be a minimizing sequence of $c_{g}(\varepsilon)$. Then

$$
\int_{\Omega}\left\{\left|\Delta \phi_{j}\right|^{2}+\frac{1}{\varepsilon^{2}}\left(1-|\nabla \phi|^{2}\right)^{2}\right\} d x=c_{g}(\varepsilon)+o(1)
$$

as $j \rightarrow \infty$. Thus $\left\{\Delta \phi_{j}\right\}$ is bounded in $L^{2}(\Omega)$ and $\left\{\nabla \phi_{j}\right\}$ is bounded in $L^{4}\left(\Omega, \mathbb{R}^{3}\right)$ and so bounded in $L^{2}\left(\Omega, \mathbb{R}^{3}\right)$. Since $\phi_{j}=\varphi_{0}$ on $\partial \Omega$, it follows from Girault and Raviart [7, p.12] that

$$
\left\|\phi_{j}\right\|_{W^{2,2}(\Omega)} \leq C\left\{\left\|\Delta \phi_{j}\right\|_{L^{2}(\Omega)}+\left\|\varphi_{0}\right\|_{H^{3 / 2}(\partial \Omega)}\right\}
$$

Hence $\left\{\phi_{j}\right\}$ is bounded in $W^{2,2}(\Omega)$. Passing to a subsequence, we may assume that $\phi_{j} \rightarrow \phi$ weakly in $W^{2,2}(\Omega)$ and strongly in $W^{1,4}(\Omega)$. Therefore we have $\phi=\varphi_{0}, \frac{\partial \phi}{\partial \boldsymbol{\nu}}=\varphi_{1}$ on $\partial \Omega$. So $\phi \in W^{2,2}\left(\Omega, \varphi_{0}, \varphi_{1}\right)$, and

$$
J_{\varepsilon}[\phi] \leq \liminf _{j \rightarrow \infty} J_{\varepsilon}\left[\phi_{j}\right]=c_{g}(\varepsilon)
$$

Hence $\phi$ is a minimizer of $c_{g}(\varepsilon)$. 
(ii) Let $\phi_{\varepsilon} \in W^{2,2}\left(\Omega, \varphi_{0}, \varphi_{1}\right)$ be a minimizer of $c_{g}(\varepsilon)$. Then

$$
c_{g}\left(\phi_{\varepsilon}\right)=\int_{\Omega}\left\{\left|\Delta \phi_{\varepsilon}\right|^{2}+\frac{1}{\varepsilon^{2}}\left(1-\left|\nabla \phi_{\varepsilon}\right|^{2}\right)^{2}\right\} d x .
$$

If $\mathbb{G}(\boldsymbol{e}) \neq \emptyset$, it follows from Proposition 1.1 (cf. [9, Lemma 5.2]) that $G(\boldsymbol{e})=$ $\inf _{\phi \in W^{2,2}(\Omega)} \int_{\Omega}|\Delta \phi|^{2} d x$ is achieved. We note that by the construction of $\varphi_{0}$ and $\varphi_{1}$, we see that $\mathbb{G}(\boldsymbol{e}) \subset W^{2,2}\left(\Omega, \varphi_{0}, \varphi_{1}\right)$. Let $\phi_{0} \in \mathbb{G}(\boldsymbol{e})$ be a minimizer of $G(e)$. Then

$$
c_{g}(\varepsilon) \leq \int_{\Omega}\left|\Delta \phi_{0}\right|^{2} d x=G(\boldsymbol{e}) \text { for any } \varepsilon>0 .
$$

Thus $\left\{c_{g}(\varepsilon)\right\}$ is bounded.

Conversely, let $c_{g}(\varepsilon) \leq C$ for any $\varepsilon>0$ where $C$ is a constant independent of $\varepsilon>0$. Then

$$
\int_{\Omega}\left\{\left|\Delta \phi_{\varepsilon}\right|^{2}+\frac{1}{\varepsilon^{2}}\left(1-\left|\nabla \phi_{\varepsilon}\right|^{2}\right)^{2}\right\} d x \leq C .
$$

Thus $\left\{\phi_{\varepsilon}\right\}$ is bounded in $W^{2,2}(\Omega)$. Passing to a subsequence, we may assume that $\phi_{\varepsilon} \rightarrow \phi$ weakly in $W^{2,2}(\Omega)$ and strongly in $W^{1,4}(\Omega)$. We can see that $\phi=\varphi_{0}, \frac{\partial \phi}{\partial \boldsymbol{\nu}}=\varphi_{1}$ on $\partial \Omega$. From $(2.7)$, we have

$$
\int_{\Omega}\left(1-\left|\nabla \phi_{\varepsilon}\right|^{2}\right)^{2} d x \leq C \varepsilon^{2} .
$$

Since $\nabla \phi_{\varepsilon} \rightarrow \nabla \phi$ strongly in $L^{4}\left(\Omega, \mathbb{R}^{3}\right)$ and in $L^{2}\left(\Omega, \mathbb{R}^{2}\right)$, letting $\varepsilon \rightarrow 0$, we see that $\int_{\Omega}\left(1-|\nabla \phi|^{2}\right)^{2} d x=0$. Thus $|\nabla \phi|=1$ a.e. in $\Omega$. Therefore, $\phi \in \mathbb{G}(\boldsymbol{e})$, so $\mathbb{G}(\boldsymbol{e}) \neq \emptyset$. From (2.6), we have

$$
\begin{aligned}
\int_{\Omega}|\Delta \phi|^{2} d x & \leq \liminf _{\varepsilon \rightarrow 0} \int_{\Omega}\left\{\left|\Delta \phi_{\varepsilon}\right|^{2}+\frac{1}{\varepsilon^{2}}\left(1-\left|\nabla \phi_{\varepsilon}\right|^{2}\right)^{2}\right\} d x \\
& =\liminf _{\varepsilon \rightarrow 0} c_{g}(\varepsilon) \\
& \leq \limsup _{\varepsilon \rightarrow 0} c_{g}(\varepsilon) \leq G(\boldsymbol{e}) .
\end{aligned}
$$

Thus $\phi$ is a minimizer of $G(\boldsymbol{e})$, and $\lim _{\varepsilon \rightarrow 0} c_{g}(\varepsilon)=G(\boldsymbol{e})$. Moreover, since

$$
\begin{aligned}
G(\boldsymbol{e}) & =\int_{\Omega}|\Delta \phi|^{2} d x \\
& \leq \liminf _{\varepsilon \rightarrow 0} \int_{\Omega}\left|\Delta \phi_{\varepsilon}\right|^{2} d x \\
& \leq \limsup _{\varepsilon \rightarrow 0} \int_{\Omega}\left|\Delta \phi_{\varepsilon}\right|^{2} d x \\
& \leq \limsup _{\varepsilon \rightarrow 0} c_{g}(\varepsilon) \\
& \leq G(\boldsymbol{e})=\int_{\Omega}|\Delta \phi|^{2} d x
\end{aligned}
$$


we have

$$
\lim _{\varepsilon \rightarrow 0} \int_{\Omega}\left|\Delta \phi_{\varepsilon}\right|^{2} d x=\int_{\Omega}|\Delta \phi|^{2} d x
$$

and

$$
\lim _{\varepsilon \rightarrow 0} \frac{1}{\varepsilon^{2}} \int_{\Omega}\left(1-\left|\nabla \phi_{\varepsilon}\right|^{2}\right)^{2} d x=0 .
$$

Since $\Delta \phi_{\varepsilon} \rightarrow \Delta \phi$ weakly in $L^{2}(\Omega)$, we see that $\Delta \phi_{\varepsilon} \rightarrow \Delta \phi$ strongly in $L^{2}(\Omega)$. Since $\phi_{\varepsilon}=\phi=\varphi_{0}$ on $\partial \Omega$, we see from the similar estimate as (2.5), that $\phi_{\varepsilon} \rightarrow \phi$ strongly in $W^{2,2}(\Omega)$. This completes the proof.

\section{More general setting}

Let $\Omega \subset \mathbb{R}^{3}$ be a bounded smooth domain (not necessary to be simplyconnected). Define

$$
W^{1,2}\left(\Omega, \mathbb{S}^{2}\right)=\left\{\boldsymbol{u} \in W^{1,2}\left(\Omega, \mathbb{R}^{3}\right) ;|\boldsymbol{u}(x)|=1 \text { a.e in } \Omega\right\},
$$

and

$$
W^{1,2}\left(\Omega, \operatorname{curl}, \mathbb{S}^{2}\right)=\left\{\boldsymbol{u} \in W^{1,2}\left(\Omega, \mathbb{S}^{2}\right) ; \operatorname{curl} \boldsymbol{u}=0 \text { a.e. in } \Omega\right\} .
$$

We note that $W^{1,2}\left(\Omega, \operatorname{curl}, \mathbb{S}^{2}\right) \cap C^{1}\left(\bar{\Omega}, \mathbb{S}^{2}\right) \neq \emptyset$. In fact, for example, the vector field

$$
\boldsymbol{u}(x)=\left(\frac{x_{1}-a_{1}}{\sqrt{\left(x_{1}-a_{1}\right)^{2}+x_{2}^{2}}}, \frac{x_{2}}{\sqrt{\left(x_{1}-a_{1}\right)^{2}+x_{2}^{2}}}, 0\right)
$$

belongs to $W^{1,2}\left(\Omega, \operatorname{curl}, \mathbb{S}^{2}\right) \cap C^{1}\left(\bar{\Omega}, \mathbb{S}^{2}\right)$. Here we choose $a_{1}$ so that $x_{1}-a_{1} \neq 0$ for any $x=\left(x_{1}, x_{2}, x_{3}\right) \in \bar{\Omega}$ (cf. Aramaki [1], [2], [3]). Assume that $\boldsymbol{e} \in$ $C^{1}\left(\partial \Omega, \mathbb{S}^{2}\right)$ satisfies

$$
\boldsymbol{e}_{\tau}=\nabla_{\tau} \psi
$$

for some $\psi \in C^{2}(\partial \Omega)$. Then we note that $\boldsymbol{e}$ has a $C^{1}$ curl-free extension. Define

$$
\widehat{\mathbb{G}}(\boldsymbol{e})=\widehat{\mathbb{G}}(\boldsymbol{e}, \Omega)=\left\{\boldsymbol{u} \in W^{1,2}\left(\Omega, \operatorname{curl}, \mathbb{S}^{2}\right) ; \boldsymbol{u}=\boldsymbol{e} \text { on } \partial \Omega\right\} .
$$

If $\widehat{\mathbb{G}}(\boldsymbol{e}) \neq \emptyset$, we set the minimizing problem

$$
\widehat{G}(\boldsymbol{e})=\widehat{\mathbb{G}}(\boldsymbol{e}, \Omega)=\inf _{\boldsymbol{u} \in \mathbb{\mathbb { G }}(\boldsymbol{e})} \int_{\Omega}(\operatorname{div} \boldsymbol{u})^{2} d x .
$$


Remark 3.1. [9] treated the set $\mathbb{G}(\boldsymbol{e})$ provided that $\Omega$ is a bounded, smooth simply-connected domain, and considered the minimizing problem (1.5)-(1.6). However if $\Omega$ is simply connected, this minimizing problem is precisely equivalent to (3.2)-(3.3). In fact, the map

$$
\mathbb{G}(\boldsymbol{e}) / \mathbb{R} \ni[\phi] \mapsto \boldsymbol{u}=\nabla \phi \in \widehat{\mathbb{G}}(\boldsymbol{e})
$$

is bijictive, and define equivalent norms. To show this, if $\Omega$ is simply-connected, for any $\boldsymbol{u} \in \widehat{\mathbb{G}}(\boldsymbol{e})$, there exists $\phi \in W^{2,2}(\Omega)$ such that $\boldsymbol{u}=\nabla \phi$. Therefore, the bijectiveness is clear. For any $d \in \mathbb{R},\|\nabla \phi\|_{W^{1,2}\left(\Omega, \mathbb{R}^{3}\right)} \leq C\|\phi+d\|_{W^{2,2}(\Omega)}$. So we have

$$
\|\nabla \phi\|_{W^{1,2}\left(\Omega, \mathbb{R}^{3}\right)} \leq\|[\phi]\|_{W^{2,2}(\Omega)} / \mathbb{R} .
$$

For any $[\phi] \in \mathbb{G}(\boldsymbol{e}) / \mathbb{R}$, we can assume that $\int_{\Omega} \phi d x=0$. By the Poincaré inequality,

$$
\|\nabla \phi\|_{L^{2}\left(\Omega, \mathbb{R}^{3}\right)} \geq c\|\phi\|_{L^{2}(\Omega)} \geq\|[\phi]\|_{L^{2}(\Omega) / R}
$$

Thus we have

$$
\|\nabla \phi\|_{W^{1,2}\left(\Omega, \mathbb{R}^{3}\right)}=\|\nabla \phi\|_{L^{2}(\Omega)}+\left\|D^{2} \phi\right\|_{L^{2}(\Omega)} \geq c_{1}\|[\phi]\|_{W^{2,2}(\Omega) / \mathbb{R}} .
$$

Lemma 3.2. Assume that $\boldsymbol{e} \in C^{1}\left(\partial \Omega, \mathbb{S}^{2}\right)$ and $\mathbb{G}(\boldsymbol{e}) \neq \emptyset$. Then $\widehat{\mathbb{G}}(\boldsymbol{e})$ is achieved in $\widehat{\mathbb{G}}(\boldsymbol{e})$.

Proof. Let $\left\{\boldsymbol{u}_{j}\right\} \subset \widehat{\mathbb{G}}(\boldsymbol{e})$ be a minimizing sequence of $\widehat{G}(\boldsymbol{e})$. Then

$$
\int_{\Omega}\left(\operatorname{div} \boldsymbol{u}_{j}\right)^{2} d x=\widehat{G}(\boldsymbol{e})+o(1)
$$

as $j \rightarrow \infty$. According to Dautray and Lions [5, p.212], for any $\boldsymbol{u} \in W^{1,2}\left(\Omega, \mathbb{R}^{3}\right)$, we have

$$
\begin{aligned}
\|\boldsymbol{u}\|_{W^{1,2}\left(\Omega, \mathbb{R}^{3}\right)} \leq C\left\{\|\boldsymbol{u}\|_{L^{2}\left(\Omega, \mathbb{R}^{3}\right)}+\right. & \|\operatorname{div} \boldsymbol{u}\|_{L^{2}(\Omega)} \\
& \left.+\|\operatorname{curl} \boldsymbol{u}\|_{L^{2}\left(\Omega, \mathbb{R}^{3}\right)}+\|\boldsymbol{u} \cdot \boldsymbol{\nu}\|_{H^{1 / 2}(\partial \Omega)}\right\}
\end{aligned}
$$

If we apply this inequality for $\boldsymbol{u}=\boldsymbol{u}_{j}$, since $\left|\boldsymbol{u}_{j}\right|=1$ a.e. in $\Omega$, curl $\boldsymbol{u}_{j}=0$ a.e. in $\Omega$ and $\boldsymbol{u}_{j}=\boldsymbol{e}$ on $\partial \Omega$, we see that $\left\{\boldsymbol{u}_{j}\right\}$ is bounded in $W^{1,2}\left(\Omega, \mathbb{R}^{3}\right)$. Passing to a subsequence, we may assume that $\boldsymbol{u}_{j} \rightarrow \boldsymbol{u}_{0}$ weakly in $W^{1,2}\left(\Omega, \mathbb{R}^{3}\right)$ and a.e. in $\Omega$. Therefore, $\left|\boldsymbol{u}_{0}\right|=1$ a.e. in $\Omega$, curl $\boldsymbol{u}_{0}=0$ a.e. in $\Omega$ and $\boldsymbol{u}_{0}=\boldsymbol{e}$ on $\partial \Omega$. Hence $\boldsymbol{u}_{0} \in \widehat{\mathbb{G}}(\boldsymbol{e})$. Since $\operatorname{div} \boldsymbol{u}_{j} \rightarrow \operatorname{div} \boldsymbol{u}_{0}$ weakly in $L^{2}(\Omega)$, we have

$$
\int_{\Omega}\left(\operatorname{div} \boldsymbol{u}_{0}\right)^{2} d x \leq \liminf _{j \rightarrow \infty} \int_{\Omega}\left(\operatorname{div} \boldsymbol{u}_{j}\right)^{2} d x=\widehat{G}(\boldsymbol{e}) .
$$

Thus $\boldsymbol{u}_{0}$ is a minimizer of $\widehat{G}(\boldsymbol{e})$. 
The minimizing problem (3.2)-(3.3) is closely related to the following problem. Define

$$
W^{1,2}(\Omega, \operatorname{curl}, \boldsymbol{e})=\left\{\boldsymbol{u} \in W^{1,2}\left(\Omega, \mathbb{R}^{3}\right) ; \operatorname{curl} \boldsymbol{u}=0 \text { a.e. in } \Omega, \boldsymbol{u}=\boldsymbol{e} \text { on } \partial \Omega\right\} .
$$

We note that if we assume that $(3.1)$ holds, then $W^{1,2}(\Omega, \operatorname{curl}, \boldsymbol{e}) \neq \emptyset$. Define a functional

$$
\widehat{J}_{\varepsilon}[\boldsymbol{u}]=\int_{\Omega}\left\{(\operatorname{div} \boldsymbol{u})^{2}+\frac{1}{\varepsilon^{2}}\left(1-|\boldsymbol{u}|^{2}\right)^{2}\right\} d x
$$

and set

$$
\widehat{c}_{g}(\varepsilon)=\inf _{\boldsymbol{u} \in W^{1,2}(\Omega, \operatorname{curl}, \boldsymbol{e})} \widehat{J}_{\varepsilon}[\boldsymbol{u}] .
$$

The main theorem in this section is as follows.

Theorem 3.3. Assume that $\boldsymbol{e} \in C^{1}\left(\partial \Omega, \mathbb{R}^{3}\right)$ satisfies (3.1). Then the following holds.

(i) $\widehat{c}_{g}(\varepsilon)$ is achieved in $W^{1,2}(\Omega$, curl, $\boldsymbol{e})$ for any $\varepsilon>0$.

(ii) $\widehat{\mathbb{G}}(\boldsymbol{e}) \neq \emptyset$ if and only if $\left\{\widehat{c}_{g}(\varepsilon)\right\}_{\varepsilon>0}$ is bounded uniformly with respect to $\varepsilon>0$.

In this case, let $\boldsymbol{u}_{\varepsilon}$ be a minimizer of $\widehat{c}_{g}(\varepsilon)$. Then there exist a subsequence $\left\{\boldsymbol{u}_{\varepsilon_{j}}\right\}$ and $\boldsymbol{u}_{0} \in \widehat{\mathbb{G}}(\boldsymbol{e})$ which is a minimizer of $\widehat{G}(\boldsymbol{e})$ such that $\boldsymbol{u}_{\varepsilon_{j}} \rightarrow \boldsymbol{u}_{0}$ strongly in $W^{1,2}\left(\Omega, \mathbb{R}^{3}\right)$ as $\varepsilon_{j} \rightarrow 0$.

Proof. (i) Fix $\varepsilon>0$ and let $\left\{\boldsymbol{u}_{j}\right\} \subset W^{1,2}(\Omega$, curl, $\boldsymbol{e})$ be a minimizing sequence of $\widehat{c}_{g}(\varepsilon)$. Then

$$
\int_{\Omega}\left\{\left(\operatorname{div} \boldsymbol{u}_{j}\right)^{2}+\frac{1}{\varepsilon^{2}}\left(1-\left|\boldsymbol{u}_{j}\right|^{2}\right)^{2}\right\} d x=\widehat{c}_{g}(\varepsilon)+o(1)
$$

as $j \rightarrow \infty$. Therefore, since $\left\{\operatorname{div} \boldsymbol{u}_{j}\right\}$ is bounded in $L^{2}(\Omega)$, curl $\boldsymbol{u}_{j}=0,\left\{\boldsymbol{u}_{j}\right\}$ is bounded in $L^{4}\left(\Omega, \mathbb{R}^{3}\right)$ and $\boldsymbol{u}_{j}=\boldsymbol{e}$ on $\partial \Omega$, it follows from (3.4) that $\left\{\boldsymbol{u}_{j}\right\}$ is bounded in $W^{1,2}\left(\Omega, \mathbb{R}^{3}\right)$. Passing to a subsequence, we may assume that $\boldsymbol{u}_{j} \rightarrow \boldsymbol{u}_{0}$ weakly in $W^{1,2}\left(\Omega, \mathbb{R}^{3}\right)$, strongly in $L^{4}\left(\Omega, \mathbb{R}^{3}\right)$ and a.e. in $\Omega$. Therefore curl $\boldsymbol{u}_{0}=0$ a.e. in $\Omega, \boldsymbol{u}_{0}=\boldsymbol{e}$ on $\partial \Omega$. Thus we see that $\boldsymbol{u}_{0} \in W^{1,2}(\Omega$, curl, $\boldsymbol{e})$. Hence we have

$$
\begin{aligned}
\int_{\Omega}\left\{\left(\operatorname{div} \boldsymbol{u}_{0}\right)^{2}+\frac{1}{\varepsilon^{2}}(1-\right. & \left.\left.\left|\boldsymbol{u}_{0}\right|^{2}\right)^{2}\right\} d x \\
& \leq \liminf _{j \rightarrow \infty} \int_{\Omega}\left\{\left(\operatorname{div} \boldsymbol{u}_{j}\right)^{2}+\frac{1}{\varepsilon^{2}}\left(1-\left|\boldsymbol{u}_{j}\right|^{2}\right)^{2}\right\} d x=\widehat{c}_{g}(\varepsilon) .
\end{aligned}
$$

Thus $\boldsymbol{u}_{0}$ is a minimizer of $\widehat{c}_{g}(\varepsilon)$. 
(ii) Let $\boldsymbol{u}_{\varepsilon} \in W^{1,2}(\Omega$, curl, $\boldsymbol{e})$ be a minimizer of $\widehat{c}_{g}(\varepsilon)$. If $\widehat{\mathbb{G}}(\boldsymbol{e}) \neq \emptyset$, we see that $\emptyset \neq \widehat{\mathbb{G}}(\boldsymbol{e}) \subset W^{1,2}(\Omega$, curl, $\boldsymbol{e})$. If we choose $\widehat{\boldsymbol{u}} \in \widehat{\mathbb{G}}(\boldsymbol{e})$ so that $\widehat{\boldsymbol{u}}$ is a minimizer of $\widehat{G}(\boldsymbol{e})$, we have

$$
\begin{aligned}
\widehat{c}_{g}(\varepsilon)=\int_{\Omega}\left\{\left(\operatorname{div} \boldsymbol{u}_{\varepsilon}\right)^{2}+\frac{1}{\varepsilon^{2}}\left(1-\left|\boldsymbol{u}_{\varepsilon}\right|^{2}\right)^{2}\right\} d x & \\
& \leq \int_{\Omega}(\operatorname{div} \widehat{\boldsymbol{u}})^{2} d x=\widehat{G}(\boldsymbol{e}) .
\end{aligned}
$$

In particular, $\left\{\widehat{c}_{g}(\varepsilon)\right\}$ is uniformly bounded with respect to $\varepsilon>0$.

Conversely, if there exists a constant $C>0$ independent of $\varepsilon>0$ such that $\widehat{c}_{g}(\varepsilon) \leq C$, then

$$
\widehat{c}_{g}(\varepsilon)=\int_{\Omega}\left\{\left(\operatorname{div} \boldsymbol{u}_{\varepsilon}\right)^{2}+\frac{1}{\varepsilon^{2}}\left(1-\left|\boldsymbol{u}_{\varepsilon}\right|^{2}\right)^{2}\right\} d x \leq C .
$$

Therefore we can see that $\left\{\operatorname{div} \boldsymbol{u}_{\varepsilon}\right\}$ is bounded in $L^{2}(\Omega)$ and $\left\{\boldsymbol{u}_{\varepsilon}\right\}$ is bounded in $L^{4}\left(\Omega, \mathbb{R}^{3}\right)$. Since $\operatorname{curl} \boldsymbol{u}_{\varepsilon}=0$ a.e. in $\Omega, \boldsymbol{u}_{\varepsilon}=\boldsymbol{e}$ on $\partial \Omega$, it follows from (3.4) that $\left\{\boldsymbol{u}_{\varepsilon}\right\}$ is bounded in $W^{1,2}\left(\Omega, \mathbb{R}^{3}\right)$. Passing to a subsequence, we may assume that $\boldsymbol{u}_{\varepsilon} \rightarrow \boldsymbol{u}_{0}$ weakly in $W^{1,2}\left(\Omega, \mathbb{R}^{3}\right)$, strongly in $L^{4}\left(\Omega, \mathbb{R}^{3}\right)$ and a.e. in $\Omega$. Thus we see that $\operatorname{curl} \boldsymbol{u}_{0}=0$ a.e. in $\Omega$ and $\boldsymbol{u}_{0}=\boldsymbol{e}$ on $\partial \Omega$. Hence $\boldsymbol{u}_{0} \in W^{1,2}(\Omega, \operatorname{curl}, \boldsymbol{e})$. By (3.6), we have

$$
\int_{\Omega}\left(1-\left|\boldsymbol{u}_{\varepsilon}\right|^{2}\right)^{2} d x \leq C \varepsilon^{2} .
$$

Since $\boldsymbol{u}_{\varepsilon} \rightarrow \boldsymbol{u}_{0}$ in $L^{4}\left(\Omega, \mathbb{R}^{3}\right)$, letting $\varepsilon \rightarrow 0$, we have $\int_{\Omega}\left(1-|\boldsymbol{u}|^{2}\right)^{2} d x=0$. Thus since $\left|\boldsymbol{u}_{0}\right|=1$ a.e. in $\Omega$, we see that $\boldsymbol{u}_{0} \in \widehat{\mathbb{G}}(\boldsymbol{e})$. Therefore, $\widehat{\mathbb{G}}(\boldsymbol{e}) \neq \emptyset$. In this case, from (3.5), we have

$$
\begin{aligned}
\int_{\Omega}\left(\operatorname{div} \boldsymbol{u}_{0}\right)^{2} d x & \leq \liminf _{\varepsilon \rightarrow 0} \int_{\Omega}\left(\operatorname{div} \boldsymbol{u}_{\varepsilon}\right)^{2} d x \\
& \leq \limsup _{\varepsilon \rightarrow 0} \int_{\Omega}\left(\operatorname{div} \boldsymbol{u}_{\varepsilon}\right)^{2} d x \\
& \leq \limsup _{\varepsilon \rightarrow 0} \widehat{c}_{g}(\varepsilon) \\
& \leq \widehat{G}(\boldsymbol{e}) .
\end{aligned}
$$

Thus $\boldsymbol{u}_{0}$ is a minimizer of $\widehat{G}(\boldsymbol{e})$ and

$$
\lim _{\varepsilon \rightarrow 0} \int_{\Omega}\left(\operatorname{div} \boldsymbol{u}_{\varepsilon}\right)^{2} d x=\int_{\Omega}\left(\operatorname{div} \boldsymbol{u}_{0}\right)^{2} d x .
$$

Moreover, since

$$
\widehat{G}(\boldsymbol{e}) \leq \liminf _{\varepsilon \rightarrow 0} \widehat{c}_{g}(\varepsilon) \leq \limsup _{\varepsilon \rightarrow 0} \widehat{c}_{g}(\varepsilon)=\widehat{G}(\boldsymbol{e}),
$$


we have

$$
\lim _{\varepsilon \rightarrow 0} \int_{\Omega} \frac{1}{\varepsilon^{2}}\left(1-\left|\boldsymbol{u}_{\varepsilon}\right|^{2}\right)^{2} d x=0
$$

Finally we use the formulas (cf. [8]): for any $\boldsymbol{u} \in W^{1,2}\left(\Omega, \mathbb{R}^{3}\right)$,

$$
\begin{aligned}
& |\nabla \boldsymbol{u}|^{2}=(\operatorname{div} \boldsymbol{u})^{2}+|\operatorname{curl} \boldsymbol{u}|^{2}+\operatorname{Tr}(\nabla \boldsymbol{u})^{2}-(\operatorname{div} \boldsymbol{u})^{2}, \\
& \operatorname{Tr}(\nabla \boldsymbol{u})^{2}-(\operatorname{div} \boldsymbol{u})^{2}=\operatorname{div}[(\nabla \boldsymbol{u}) \boldsymbol{u}-(\operatorname{div} \boldsymbol{u}) \boldsymbol{u}], \\
& \int_{\Omega}\left\{\operatorname{Tr}(\nabla \boldsymbol{u})^{2}-(\operatorname{div} \boldsymbol{u})^{2}\right\} d x=\int_{\partial \Omega}\left[\left(\nabla_{\tan } \boldsymbol{u}\right) \boldsymbol{u}-\operatorname{Tr}\left(\nabla_{\tan } \boldsymbol{u}\right) \boldsymbol{u}\right] \cdot \boldsymbol{\nu} d S
\end{aligned}
$$

where $\nabla_{\tan } \boldsymbol{u}=\nabla \boldsymbol{u}-(\nabla \boldsymbol{u}) \boldsymbol{\nu} \otimes \boldsymbol{\nu}$ depends only on $\left.\boldsymbol{u}\right|_{\partial \Omega}$ and the first order tangential derivatives of $\left.\boldsymbol{u}\right|_{\partial \Omega}$. Using these formulas with $\boldsymbol{u}=\boldsymbol{u}_{\varepsilon}$, we have

$$
\lim _{\varepsilon \rightarrow 0} \int_{\Omega}\left|\nabla \boldsymbol{u}_{\varepsilon}\right|^{2} d x=\int_{\Omega}|\nabla \boldsymbol{u}|^{2} d x
$$

Since $\boldsymbol{u}_{\varepsilon} \rightarrow \boldsymbol{u}$ weakly in $W^{1,2}\left(\Omega, \mathbb{R}^{3}\right)$ and strongly in $L^{2}\left(\Omega, \mathbb{R}^{3}\right)$, we see that $\boldsymbol{u}_{\varepsilon} \rightarrow \boldsymbol{u}$ strongly in $W^{1,2}\left(\Omega, \mathbb{R}^{3}\right)$.

\section{Auxiliary variational problem}

In this section we consider the auxiliary variational problem under the divergencefree setting instead of the curl-free setting.

Define

$$
\mathbb{R}(\boldsymbol{e})=\mathbb{R}(\boldsymbol{e}, \Omega)=\left\{\boldsymbol{u} \in W^{1,2}\left(\Omega, \mathbb{S}^{2}, \boldsymbol{e}\right) ; \operatorname{div} \boldsymbol{u}=0 \text { a.e. in } \Omega\right\}
$$

If $\mathbb{R}(\boldsymbol{e}) \neq \emptyset$, we set

$$
R(\boldsymbol{e})=R(\boldsymbol{e}, \Omega)=\inf _{\boldsymbol{u} \in \mathbb{R}(\boldsymbol{e})} \int_{\Omega}|\operatorname{curl} \boldsymbol{u}|^{2} d x .
$$

The problem is closely related to the following problem. Let

$$
W^{1,2}(\Omega, \operatorname{div}, \boldsymbol{e})=\left\{\boldsymbol{u} \in W^{1,2}\left(\Omega, \mathbb{R}^{3}\right) ; \operatorname{div} \boldsymbol{u}=0 \text { a.e. in } \Omega, \boldsymbol{u}=\boldsymbol{e} \text { on } \partial \Omega\right.
$$

We set

$$
J_{\varepsilon}^{*}[\boldsymbol{u}]=\int_{\Omega}\left\{|\operatorname{curl} \boldsymbol{u}|^{2}+\frac{1}{\varepsilon^{2}}\left(1-|\boldsymbol{u}|^{2}\right)^{2}\right\} d x
$$

and

$$
c_{r}(\boldsymbol{e})=\inf _{\boldsymbol{u} \in W^{1,2}(\Omega, \operatorname{curl}, \boldsymbol{e})} J_{\varepsilon}^{*}[\boldsymbol{u}] .
$$

Similarly as Lemma 3.2 (cf. Proposition 1.2 or [9, Lemma 4.3]), we have 
Lemma 4.1. If $\mathbb{R}(\boldsymbol{e}) \neq \emptyset$, then $R(\boldsymbol{e})$ is achieved in $\mathbb{R}(\boldsymbol{e})$.

Definition 4.2. Let $\boldsymbol{e} \in H^{1 / 2}(\partial \Omega)$. We say that $\boldsymbol{e}$ has a divergence-free extension if there exists $\boldsymbol{u} \in W^{1,2}\left(\Omega, \mathbb{R}^{3}\right)$ such that $\operatorname{div} \boldsymbol{u}=0$ a.e. in $\Omega$ and $\boldsymbol{u}=\boldsymbol{e}$ on $\partial \Omega$.

Then we can prove the following lemma.

Lemma 4.3. Let $\Omega$ be a smooth bounded domain in $\mathbb{R}^{3}$ and let $e \in H^{1 / 2}(\partial \Omega)$. Then $\boldsymbol{e}$ has a divergence-free extension if and only if

$$
\int_{\partial \Omega} \boldsymbol{e} \cdot \boldsymbol{\nu} d S=0
$$

Proof. If there exists $\boldsymbol{u} \in W^{1,2}\left(\Omega, \mathbb{R}^{3}\right)$ such that $\operatorname{div} \boldsymbol{u}=0$ a.e. in $\Omega$ and $\boldsymbol{u}=\boldsymbol{e}$ on $\partial \Omega$, then it is easily seen from the Green theorem that

$$
\int_{\partial \Omega} \boldsymbol{e} \cdot \boldsymbol{\nu} d S=\int_{\Omega} \operatorname{div} \boldsymbol{u} d x=0 .
$$

Conversely, assume that (4.6) holds. Choose $\boldsymbol{v} \in W^{1,2}\left(\Omega, \mathbb{R}^{3}\right)$ such that $\boldsymbol{v}=\boldsymbol{e}$ on $\partial \Omega$. Then

$$
\int_{\Omega} \operatorname{div} \boldsymbol{v} d x=\int_{\partial \Omega} \boldsymbol{v} \cdot \boldsymbol{\nu} d S=\int_{\partial \Omega} \boldsymbol{e} \cdot \boldsymbol{\nu} d S=0 .
$$

By $[5$, p.233], we have

$$
\operatorname{div} W_{0}^{1,2}\left(\Omega, \mathbb{R}^{3}\right)=\left\{\phi \in L^{2}(\Omega) ; \int_{\Omega} \phi d x=0\right\} .
$$

Therefore, we can choose $\boldsymbol{w} \in W_{0}^{1,2}\left(\Omega, \mathbb{R}^{3}\right)$ such that $\operatorname{div} \boldsymbol{w}=\operatorname{div} \boldsymbol{v}$ a.e. in $\Omega$. If we put $\boldsymbol{u}=\boldsymbol{v}-\boldsymbol{w}$, then $\operatorname{div} \boldsymbol{u}=0$ a.e. in $\Omega$ and $\boldsymbol{u}=\boldsymbol{e}$ on $\partial \Omega$.

Similarly as Theorem 3.3, we can get the following.

Theorem 4.4. Assume that $\boldsymbol{e} \in H^{1 / 2}\left(\partial \Omega, \mathbb{R}^{3}\right)$ satisfies (4.6). Then the following holds.

(i) $\widehat{c}_{r}(\varepsilon)$ is achieved in $W^{1,2}(\Omega$, div, e for any $\varepsilon>0$.

(ii) $\widehat{\mathbb{R}}(\boldsymbol{e}) \neq \emptyset$ if and only if $\left\{\widehat{c}_{r}(\varepsilon)\right\}_{\varepsilon>0}$ is bounded uniformly with respect to $\varepsilon>0$.

In this case, let $\boldsymbol{u}_{\varepsilon}$ be a minimizer of $\widehat{c}_{r}(\varepsilon)$. Then there exist a subsequence $\left\{\boldsymbol{u}_{\varepsilon_{j}}\right\}$ and $\boldsymbol{u}_{0} \in \widehat{\mathbb{R}}(\boldsymbol{e})$ which is a minimizer of $\widehat{R}(\boldsymbol{e})$ such that $\boldsymbol{u}_{\varepsilon_{j}} \rightarrow \boldsymbol{u}_{0}$ strongly in $W^{1,2}\left(\Omega, \mathbb{R}^{3}\right)$ as $\varepsilon_{j} \rightarrow 0$. it.

Since the proof of Theorem 4.4 is parallel to that of Theorem 3.3, we omit 


\section{References}

[1] Aramaki, J., The effect of external fields in the theory of liquid crystals, Tokyo J. Math., Vol. 35, No. 1, (2012), 181-211.

[2] Aramaki, J., The Freedericksz transition and the asymptotic behavior in nematic liquid crystals, J. Partial Diff. Equa., Vol. 25, No. 3, (2012), 276-294.

[3] Aramaki, J., Magnetic field-induced stability of a specific configuration and the asymptotic behavior of minimizers in nematic liquid crystals, Turkish J. Math., Vol. 37, No. 6, (2013), 1001-1021.

[4] Aramaki, J., Variational problems associated with smectic-C to nematic or smectic-A to nematic phase transition in the theory of liquid crystals, Far East J. Math. Sci., Vol. 41, No. 2, (2010), 153-198.

[5] Dautray, R. and Lions, J. L., Mathematical Analysis and Numerical Method for Science and Technology Vol. 3, Springer Verlag, New York, (1990).

[6] de Gennes, P. G. and Prost, J., The physics of Liquid Crystals, Second edition, Oxford, Oxford Science Publications, 1993.

[7] Girault, V. and Raviart, P. A., Finite Element Methods for Navier-Stokes Equations, Springer, Berlin, Heidelberg, New York, Tokyo, 1986.

[8] Hardt, R, Kinderlehrer, D. and Lin, F- H., Existence and partial regularity of static liquid crystal configurations, Commun. Math. Phys., Vol. 105, (1986), 547-570.

[9] Pan, X. -B., Landau-de Gennes model of liquid crystals and critical wave number, Comm. Math. Phys., Vol. 239, (2003), 343-382.

[10] Pan, X. -B. and Qi, Y., Asymptotics of minimizers of variational problems involving curl functional, J. Math. Phys., Vol. 41, No. 7, (2000), 50335063.

Receivd: November 23, 2013 